\title{
Adaptation of Thalamic Neurons Provides Information about the Spatiotemporal Context of Stimulus History
}

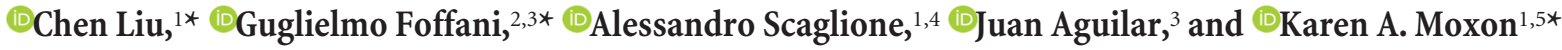 \\ ${ }^{1}$ School of Biomedical Engineering, Science and Health System, Drexel University, Philadelphia, Pennsylvania 19104, ${ }^{2} \mathrm{CINAC}$, University Hospital HM \\ Puerta del Sur, CEU-San Pablo University, Móstoles, 28938 Madrid, Spain, ${ }^{3}$ Hospital Nacional de Parapléjicos, SESCAM, 45071 Toledo, Spain, ${ }^{4}$ Neural \\ Circuits and Cognition Unit, Laboratory of Behavioral Neuroscience, National Institute on Aging, National Institutes of Health, Baltimore, Maryland 21224, \\ and ${ }^{5}$ Department of Biomedical Engineering, University of California, Davis, Davis, California 95616
}

Adaptation of neural responses due to the history of sensory input has been observed across all sensory modalities. However, the computational role of adaptation is not fully understood, especially when one considers neural coding problems in which adaptation increases the ambiguity of the neural responses to simple stimuli. To address this, we quantified the impact of adaptation on the information conveyed by thalamic neurons about paired whisker stimuli in male rat. At the single neuron level, although paired-pulse adaptation reduces the information about the present stimulus, the information per spike increases. Moreover, the adapted response can convey significant amounts of information about whether, when and where a previous stimulus occurred. At the population level, ambiguity of the adapted responses about the present stimulus can be compensated for by large numbers of neurons. Therefore, paired-pulse adaptation does not reduce the discriminability of simple stimuli. It provides information about the spatiotemporal context of stimulus history.

Key words: encoding; in vivo; sensory coding; somatosensory; vibrissae; whiskers

\section{Significance Statement}

The present work provides a computational framework that demonstrates how adaptation allows neurons to encode spatiotemporal dynamics of stimulus history.

\section{Introduction}

Somatosensory processing is an active process that allows the brain to acquire information from the environment by passing the sensory organs such as fingers or whiskers along objects. Understanding how the neuronal circuits integrate these stimuli into useful sensory information is a fundamental question in systems neuroscience. It is well established that the responses to repetitive stimuli, within tens of milliseconds to seconds, are attenuated or adapted (Chung et al., 2002; Hollins et al., 2011) and that this adaptation occurs in all sensory modalities and in both cortical

Received March 7, 2017; revised Aug. 2, 2017; accepted Aug. 16, 2017.

Author contributions: C.L., G.F., A.S., J.A., and K.A.M. designed research; A.S., J.A., and K.A.M. performed research; C.L., G.F., A.S., and K.A.M. analyzed data; C.L., G.F., A.S., J.A., and K.A.M. wrote the paper.

This work was supported by R01NS096971 from the National Institute of Health, CBET-1402984 from the National Science Foundation, and Grant 89500 from the Shriners Hospital for Children.

The authors declare no competing financial interests.

*C.L., G.F., and K.A.M. contributed equally to this work.

Correspondence should be addressed to either of the following: Karen A. Moxon, Drexel University, 3141 Chestnut Street, Philadelphia, PA 19104, E-mail: moxon@ucdavis.edu; or Guglielmo Foffani, CINAC, University Hospital HM Puerta del Sur, CEU-San Pablo University, Móstoles, 28938 Madrid, Spain, E-mail: gfoffani.hmcinac@hmhospitales.com.

D0I:10.1523/JNEUROSCI.0637-17.2017

Copyright $(2017$ the authors $\quad 0270-6474 / 17 / 3710012-10 \$ 15.00 / 0$ and subcortical areas (Laughlin, 1989; Higley and Contreras, 2007; Wark et al., 2007; Gutnisky and Dragoi, 2008; Anderson et al., 2009; Haith et al., 2009; Chase et al., 2012; Farkhooi et al., 2013). For single neurons specifically, adaptation has been generally characterized in terms of decreased firing rate (Chung et al., 2002) or modification of tuning curves (Butts and Goldman, 2006). These changes are produced by several mechanisms at the cellular-to-circuit level, including synaptic depression and recurrent and postsynaptic inhibition (Chung et al., 2002; CastroAlamancos, 2004; Higley and Contreras, 2007). Nonetheless, the computational role of adaptation during touch, when the brain must continuously decode the dynamics of when and where a stimulus occurred to identify attributes of an object (e.g., shape, texture) is not completely clear.

For relatively complex continuous stimuli, adaptation may enhance information transmission by rescaling the neuronal responses to match the statistical distribution of the inputs (Barlow, 1961; Fairhall et al., 2001; Dean et al., 2005; Maravall et al., 2007). Rescaling would allow the brain to efficiently encode stimuli whose statistics vary in time across multiple timescales (Wark et al., 2007), and react to novel events (Ranganath and Rainer, 2003). However, this form of efficient coding does not explicitly 
address the following intuitive problem that arises for simple stimuli: given that the magnitude of a single neuron's response provides a major source of information about the stimulus (e.g., stimulus location or stimulus intensity), adaptation seems to increase the ambiguity of the response, limiting the information conveyed about the stimulus. Within this computational framework, here we hypothesized that the loss of information about the current stimulus is the price paid by neurons to gain information about the short-term history of the stimuli.

We tested this hypothesis by focusing on the problem of discriminating stimulus location. Specifically, we used a pairedstimulus paradigm to assess the effect of repetitive stimuli (with 50 and $100 \mathrm{~ms}$ interstimulus interval), to the same location or across two different locations on the whisker pad, on the response of thalamic neurons in anesthetized rats. Using information theoretical analysis, we assessed the impact of paired-pulse adaptation on the capacity of single neurons and populations to convey information about the location of the current stimulus and to represent contextual (spatial and temporal) information about the stimulus in the past. Our results suggest that although pairedpulse adaptation may increase the uncertainty about the location of the second of two paired stimuli (i.e., the current stimulus), it allows contextual information about the previous stimulus to be retained in the response to the current stimulus. The ambiguity in the information at the single-neuron level about the current stimulus location can be compensated for by large populations of neurons.

\section{Materials and Methods}

Overview. This dataset of single neuron recordings was collected from six male Wistar rats $(250-350 \mathrm{~g})$, anesthetized with urethane $(1.5 \mathrm{~g} / \mathrm{kg}$, stage III-3), simultaneously with previously published data (Scaglione et al., 2011), with some overlap between the datasets. Briefly, wires were implanted into the whisker pad of the animal and single neurons were recorded from the ventro-posteromedial (VPM) nucleus of the thalamus while pairs of stimuli were applied to the whisker pad (Fig. 1). Changes in the single-trial responses to the paired stimuli were compared and information theoretic analysis was applied to understand the effect of pairedpulse adaptation on the representation of information about the stimuli. All experiments were performed following the rules of the International Council for Laboratory Animal Science, European Union regulation 86/609/EEC.

Whisker pad stimulation. Before starting the surgery for the electrophysiological recordings, eight electrical whisker stimulators were implanted into the whisker pad using methods similar to our previous work (Moxon et al., 2009). The rationale for using electrical stimuli instead of mechanical stimuli was to minimize any contribution of direction selectively on the information conveyed about stimulus location. Each stimulator consisted of a Teflon-insulated twisted pair of stainless steel wires (California Wire). Insulation was stripped on one end of the wires for 1 $\mathrm{mm}$, and the two wires were then offset by $2-3 \mathrm{~mm}$ to avoid short circuit and bent to form a hook that served to anchor the stimulator at the base of the specific whisker. A small incision $(1 \mathrm{~cm})$ was made on the left side of the face $\sim 2 \mathrm{~cm}$ caudal to the vibrissal area. Then, a regular 30 -gauge needle was used to tunnel each whisker-pad stimulator under the skin to a specific whisker. The whisker-pad stimulators were anchored to eight whiskers $[\mathrm{A}(2,4), \mathrm{C}(1,3,5)$, and $\mathrm{E}(1,3,5)]$, so that there was always at least one whisker that separated each stimulator in all directions. The final position of each stimulator was checked by sending short pulses of current $(50 \mu \mathrm{s})$ through the specific whisker pad to elicit muscle activation. For each stimulator, the intensity of the pulses $(0.2-1 \mathrm{~mA})$ was adjusted so that the corresponding whisker moved $\sim 2 \mathrm{~mm}$ at $5 \mathrm{~mm}$ from the base in any direction. To ensure that no other whiskers were moving, the stimulated whisker was observed under magnification using a stereomicroscope (LEICA M300; Leica Microsystems). Finally, a suture point near the original incision on the face was used to avoid relative move-

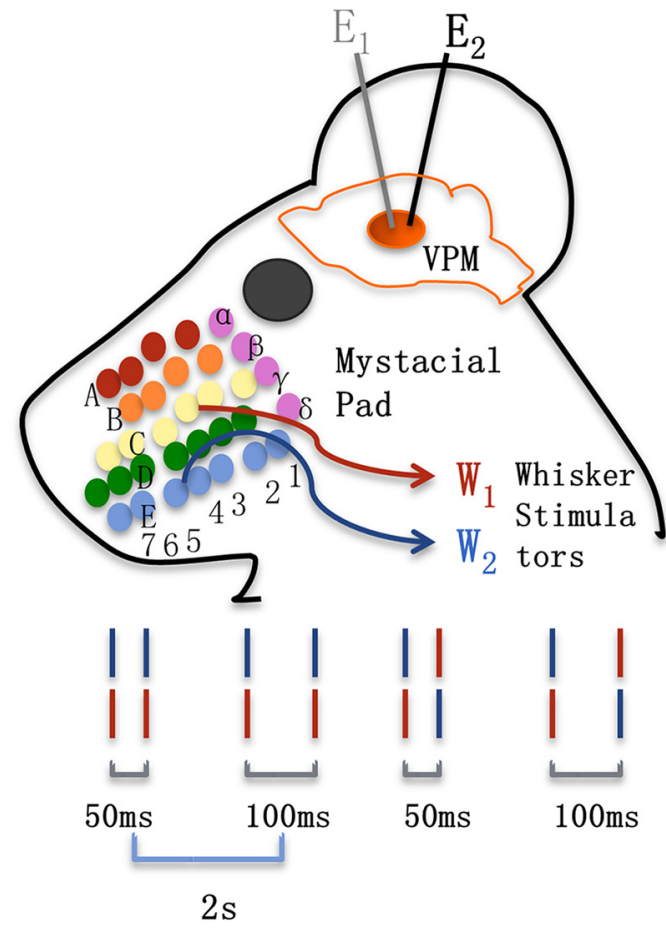

Figure 1. Experimental procedure. Single-neuron responses were collected with two highimpedance tungsten electrodes E1 (gray) and E2 (black) lowered into the VPM nucleus of the thalamus. Paired stimuli were delivered to whisker stimulators $W_{1}$ (red) and $W_{2}$ (blue). The protocol included either same-whisker stimulation (i.e., repetitive in the same whisker with 50 or 100 ms interstimulus interval) or cross-whisker stimulation (i.e., cross from one whisker to the other with 50 or $100 \mathrm{~ms}$ interstimulus interval). Pairs of stimuli were separated with a $2 \mathrm{~s}$ interval for neurons to recovery from adaptation before proceeding to the next trial, with 200 trials per condition.

ment of the stimulator with respect to the face. The above procedure was repeated until all eight stimulators were implanted. The rationale of using pulses of short duration ( $50 \mu \mathrm{s})$ was to reproduce impulsive stimuli, to maximize the overall responsiveness of VPM neurons, which can be selective to different kinetic features of whisker stimuli.

Neuronal data acquisition. To test the effects of paired-pulse adaptation on the representation of information, a protocol of paired electrical stimuli was applied to the whisker pad and single neurons were recorded from the thalamus (Fig. 1). After all whisker-pad stimulators were implanted and the wire ends secured in place, the animal's head was fixed in a stereotaxic frame (SR-6R; Narishige Scientific Instruments). Craniotomies were drilled over the right hemisphere above the VPM nucleus at -3 to $-5 \mathrm{~mm}$ anteroposterior and $3-5 \mathrm{~mm}$ mediolateral from bregma. Pairs of VPM neurons were recorded simultaneously by using two high impedance tungsten electrodes $(2-4 \mathrm{M} \Omega$ at $1 \mathrm{kHz})$. Electrodes were slowly and independently lowered down to the VPM (4-6 mm ventrodorsal from the pial surface) at a speed of $100 \mu \mathrm{m} / \mathrm{min}$ by means of hydraulic micromanipulators (Narishige Scientific Instruments). The continuous signal recorded at the electrode was band-passed $(200 \mathrm{~Hz}$ to $7 \mathrm{kHz}$ ), amplified (Neurolog, Digitimer), digitized at $20 \mathrm{kHz}$ (CED), and stored for off-line analysis. The digitized signal was shown on a computer screen connected to a CED system, using Spike 2 software (v5.03; CED). Electrical activity from each of the electrodes was monitored on the screen during the descent to the VPM.

Stimulation protocol. If at least two single neurons were isolated from the electrodes, a stimulation protocol was performed, using two of the eight whisker-pad stimulators $\left(\mathrm{W}_{1}\right.$ and $\mathrm{W}_{2} ;$ Fig. 1). The whisker-pad stimulators used were selected so that each stimulator was required to activate at least one of the neurons while minimizing the artifact due to electrical stimulation. Paired-pulse electrical stimuli were then delivered to the same whisker-pad stimulator or between the two stimulators, with 50 or 100 ms interstimulus interval. Each paired-pulse electrical stimu- 
lation constituted a single trial and was separated from the next one with a $2 \mathrm{~s}$ interval plus an average random interval of $200 \mathrm{~ms}$ to allow neurons to recover from adaptation before the next trial. A total of 200 trials were performed for each paired-pulse stimulation. Once the protocol was completed, the electrodes were moved to identify additional pairs of neurons. All stimuli were generated using a Master-8 electrical stimulator (AMPI), with ISO-Flex stimulus isolators (AMPI).

Single-neuron discrimination. Neurons were discriminated off-line using Plexon off-line software. Spikes were extracted from the raw signal by setting a threshold of at least $6 \times$ the SD of the amplitude of the analog signal. Discrimination between single neurons was meticulously achieved with off-line analysis, on the basis of the shape of the action potentials, using voltage threshold methods, spike sorting protocols, and template matching algorithms in a complementary way.

Neurophysiological analysis. A total of 48 neurons were discriminated from six animals. For each neuron, a peristimulus time histogram (PSTH) was generated to evaluate the response magnitude, defined as the average number of spikes (spikes/stimulus) in a $40 \mathrm{~ms}$ poststimulus window. To ensure the physiological significance of the responses, only neurons that had a significant response to the first stimulus in the pair were considered. A neuron was considered responsive if the response magnitude was greater than three times the background firing rate in a window equivalent to the poststimulus window (both expressed in spikes per stimulus). Of the 48 neurons recorded, 40 cells fulfilled this requirement and subsequent analysis is based on this set of 40 neurons. As each protocol has two whiskers stimulated, we designated the location that elicited the greater response magnitude as the primary whisker (PW), whereas the other location was the secondary whisker (SW). Under the paired-pulse adaptation protocol, the adapted response to the PW was compared across four conditions: (1) ${ }_{p w}^{50} P W$ and (2) ${ }_{p w}^{100} P W$, which represented the adapted PW response when there was a preceding PW stimulus $50 \mathrm{~ms}$ or $100 \mathrm{~ms}$ earlier, and (3) ${ }_{s w}^{50} \mathrm{PW}$ and (4) ${ }_{s w}^{100} \mathrm{PW}$ indicated the adapted PW response when the preceding stimulus was given at a SW 50 or $100 \mathrm{~ms}$ earlier. To track the discriminability of current stimulus location during adaptation, we also included the non-adapted SW response.

The response magnitude was measured to understand the impact of paired-pulse adaptation on the responsiveness of neurons. Furthermore, to characterize the trial-to-trial response variability, the Fano factor (FF; i.e., the ratio of the variance to the mean response magnitude across trials) was measured and compared. For a Poisson process, FF is exactly equal to 1 ; however, for noisy neural data, the FF is only close to 1 and asymptotically follows a Gamma distribution when it is sampled from a Poisson process (Eden and Kramer, 2010). For the experiments performed here with 200 trials per paired stimuli, the Poisson sample distribution of FF would follow a gamma distribution with shape parameters 99.5 and scale parameters 0.01 , such that the $95 \%$ confidence interval would be in the range of $0.81-1.21$. We thus divided, the neuronal responses into three regimes: sub-Poisson with FF $<0.81$, super-Poisson with FF $>1.21$, and nearly Poisson otherwise. Then, a $\chi^{2}$ analysis was used to study the effect of paired-pulse adaptation on the distribution of neuronal responses across different Poissonian regimes.

Information theoretic analysis. To understand the effect of paired-pulse adaptation on the representation of information about the stimuli, we quantified Shannon's mutual information between stimuli $S$ and the neural responses $R$ :

$$
I(R, S)=\sum_{s \in S} \sum_{r \in S} P(r, s) \log _{2} \frac{P(r, s)}{P(r) P(s)}=H(R)-H(R \mid S),
$$

where $P(s)$ is the prior probability of the stimulus $s, P(r, s)$ is the joint probability of observing the response $r$ given the stimulus $s$, and $P(r)$ is the probability of observing the response $r$ across all stimuli. A quadratic extrapolation method was applied for the bias correction in the mutual information analysis (Magri et al., 2009). Mutual information quantifies the information (reduction of uncertainty) about the stimulus provided by the response. If the stimuli and the responses are independent from each other the mutual information is 0 , whereas if they are perfectly correlated the mutual information equals the entropy of the stimuli. In the binary discrimination problem performed here (i.e., discrimination between two equally distributed stimuli), the entropy of the stimuli is 1 bit, which represents the upper bound on the possible information conveyed.

The mutual information is a general representation of the total information conveyed by the neural response. To understand explicitly how trial-to-trial variability, sometimes interpreted in terms of noise, could be used to provide information, the general Poisson exact breakdown of the mutual information was used to calculate autocorrelation information, i.e., information exclusively contributed by spike count variability (Scaglione et al., 2010, 2011):

$$
I_{\text {auto }}(R, S)=I(R, S)-\tilde{I}(\tilde{R}, S)
$$

where the $\tilde{I}(\tilde{R}, S)$ is estimated by substituting the entropy of recorded response with that of equivalent Poisson response, i.e., the response that followed the Poisson distribution with mean equal to the average number of spike in the poststimulus time window. The Poisson firing regime represents a response in the absence of count autocorrelation, and thus all spikes fire independently such that a spike would not influence the probability of firing a subsequent spike. Therefore, subtracting the information of the non-autocorrelated response, we would get an estimation of the information exclusively presented by the spike count autocorrelation.

The capacity of single neurons to transmit information is limited and the brain could take advantage of large populations of neurons to reliably convey information. To understand the effect of paired-pulse adaptation on the capacity of a population of neurons to transfer information, we also measured the information carried by the pooled neuron responses. Pooling neurons from different animals and protocols is justified by the fact that the informational contribution of noise correlations in these experimental conditions is negligible (Scaglione et al., 2011). Information from the pooled neuron responses could be conveyed either by a count of the total number of spikes produced by multiple neurons (i.e., ignoring the heterogeneity among neurons), or by taking into account the identity of individual neuron that fire spikes in response to each stimulus (i.e., exploiting the heterogeneity among neurons). When considering only the total spikes of neurons, the direct mutual information could be applied. However, when maintaining the identity of the neuron that fired, the possible response repertoire becomes larger as the number of neurons grows and it is therefore not possible to get an accurate estimate of the probability of a response from the finite number of experimental trials performed in this study. To avoid this problem, we first used the PSTH-based classification method (Foffani and Moxon, 2004) to classify population responses $r$ into the predicted stimuli $\hat{s}(r)$, generating a coarser estimation $P(\hat{s}(r), s)$ of the full stimulus-response joint distribution $P(r, s)$ (Foffani et al., 2004, 2009; Nelken et al., 2005). Then the mutual information from this confusion matrix was calculated as a conservative estimation of population information:

$$
I(\hat{S}(R), S)=\sum_{s} \sum_{\hat{S}} P(\hat{s}(r), s) \log _{2} \frac{P(\hat{s}(r), s)}{P((\hat{s}(r)) P(s))},
$$

where the estimate $\hat{s}(r)$ is a best prediction of the stimulus based on the PSTH-based decoder, $P(\hat{s}(r), s)$ is the joint probability of the prediction given the actual stimulus, and $P(\hat{s}(r))$ is the probability of prediction regardless the actually delivered stimulus. Information was either expressed as raw total information or normalized as information per spike (Adibi et al., 2013b).

Statistical analysis. Unless specified, the general statistics performed on physiological and information measurements are one-way repeatedmeasures ANOVA under the factor of adaptation level, followed by Dunnett post hoc tests. A two-way repeated-measures ANOVA was applied on the information to detect the existence of a previous stimulus, considering the factors of paired-stimulus condition (same whisker stimulus or cross whisker stimuli) and the interstimulus-interval (50 or $100 \mathrm{~ms}$ ). Pearson's correlation coefficient was reported for pairwise correlation between measures. The results are reported in the text as mean $\pm \mathrm{SD}$, and were considered statistically significant at $p<0.05$. 


\section{Results}

\section{Neurophysiological properties of the adapted response to} paired stimuli

We analyzed a dataset of 40 VPM neurons responding to stimulation of two whiskers (Fig. 2A): the PW, which produced the greatest response (response magnitude $0.56 \pm 0.33$ spikes/stimulus, FF $0.69 \pm 0.31$, first-spike latency $6.1 \pm 4.5 \mathrm{~ms}$, first-spike jitter $2.4 \pm 2.5 \mathrm{~ms}$ ) and the SW (response magnitude $0.15 \pm 0.22$ spikes/stimulus, FF $1.04 \pm 0.43$, first-spike latency $10.2 \pm 8.5 \mathrm{~ms}$, first-spike jitter $4.7 \pm 4.1 \mathrm{~ms}$; Scaglione et al., 2011). To understand the effect of a previous stimulus on the response to the PW, we compared the non-adapted response to both the PW and the SW to that of four adapted responses: (1) ${ }_{p w}^{50} P W$ and (2) ${ }_{p w}^{100} \mathrm{PW}$ which represented the adapted PW response when there was a preceding PW stimulus 50 or $100 \mathrm{~ms}$ earlier, and (3) ${ }_{s w}^{50} \mathrm{PW}$ and (4) ${ }_{s w}^{100} \mathrm{PW}$ representing the adapted PW response when the previous stimulus was given at a SW 50 or $100 \mathrm{~ms}$ prior (Fig. $2 \mathrm{~A}$, top). The adapted responses of the SW (i.e., ${ }_{s w}^{50} S W,{ }_{s w}^{100} S W,{ }_{p w}^{50} S W$ and ${ }_{p w}^{100} S W$ Fig. $2 A$, bottom) will not be further considered, so we will generally refer to adapted PW responses.

As expected, adaptation attenuated the magnitude of the response to whisker stimulation in the thalamus $\left(F_{(5,195)}=13.71\right.$, $p<0.0001$; Fig. $2 A, B)$, depending on both the interstimulus interval between the paired stimuli and on the location of the conditioning stimulus (Fig. $2 B$ ). These data are consistent with previous reports for same whisker (Castro-Alamancos, 2004; Ganmor et al., 2010; Stüttgen and Schwarz, 2010) or crosswhisker adaptation (Lavallée and Deschênes, 2004; Higley and Contreras, 2005, 2007). At the same time, adaptation increased the response latency (up to $12.1 \pm 7.4 \mathrm{~ms}$ for ${ }_{p w}^{50} \mathrm{PW}$ ) and the trial-to-trial variability of VPM neurons, as measured by the FF $\left(F_{(5,180)}=10.65, p<0.0001\right.$; Fig. $\left.2 C\right)$. In fact, the FF exhibited a negative correlation with response magnitude $(R=-0.44, p<$ 0.0001; Fig. 2D). The more the response was adapted, the more likely it was for a neuron to transition from a less variable subPoisson regime to a more variable Poisson or supra-Poisson regime $\left(\chi_{(8)}^{2}=27.44, p<0.0001\right.$; Fig. $\left.2 E\right)$. The impact of these differences between adapted and non-adapted responses for the coding of stimulus location is examined in the following sections with specific focus on spike-count coding.

\section{Information about stimuli in the present conveyed by the adapted response}

Given that the adapted PW responses were more similar in magnitude and trial-to-trial variability to SW responses, we first evaluated the effect of this adaptation on the ability of single neurons to convey information about the stimulus; or more specifically, do the responses of single neurons allow an ideal decoder to discriminate stimulus location ( $\mathrm{PW}$ vs SW) when the response to the PW is adapted? As expected from the reduction in response magnitude, the ability to discriminate stimulus location was attenuated the more the PW response was adapted $\left(F_{(4,156)}=3.12\right.$, $p<0.0167)$. The spike count information and the difference in magnitude between the adapted PW response and the SW response were highly correlated $(R>0.93, p>0.0001$ for all groups; Fig. 3A).

Because adaptation also modulated the trial-to-trial variability of the response, we next examined the impact of adaptation on autocorrelation information, which we previously showed to significantly contribute to the total information about stimulus location (Scaglione et al., 2011). Indeed, adaptation reduced the amount of autocorrelation information $\left(F_{(4,144)}=2.87, p<0.0250\right)$. Notably, unlike the relationship between differences in response magnitude and total information, the relative proportion of autocorrelation information to the total information was greatest when the difference between the magnitudes of the responses was the least (Fig. $3 B$ ). This result suggests that differences in response variability, as measured by differences in the FF between adapted PW responses and SW responses, were increasingly responsible for conveying information about stimulus location when the differences between the response magnitudes were small.

\section{Information about stimuli in the past conveyed by the adapted response}

Encoding somatosensory information by the brain is not a stationary process that only considers current events but is instead a dynamic process that is concerned with integrating information from the past. How the brain facilitates this is unclear. To determine whether the adapted response, despite its reduced information about the current stimulus, conveys information about the previous stimulus, we first examined the information about the existence of a prior stimulus by discriminating the PW response ("a previous stimulus did not occur") from the adapted PW response ("a previous stimulus did occur"). Not surprisingly, there was significant information to detect that a prior stimulus occurred, which depended on both the location (same-whisker ${ }_{p w} P W$ vs cross-whisker $\left.{ }_{s w} P W, F_{(1,39)}=40.05, p<0.0001\right)$ and timing of the previous stimulus ( $50 \mathrm{vs} 100 \mathrm{~ms} ; F_{(1,39)}=15.82, p=$ 0.0003; Fig. $4 A$ ). This information was positively correlated to differences between the magnitude of the responses to the two stimuli $(R>0.86, p<0.0001$, data not shown), suggesting the information was mostly due to the attenuation of the physiological response. Again, when there was little difference in the magnitude of the responses, the difference in the response variability was especially critical to conveying information (Fig. 4B).

To further understand the contextual information about the previous stimuli, we first examined the capacity of neurons to discriminate the location of the previous stimulus ( $\mathrm{PW}$ or SW) given the adapted PW response to the current stimulus (Fig. 4C). The adapted PW response provided similar information about stimulus location in the past $\left(F_{(2,78)}=40.05, p=0.1811\right)$ independently of whether the previous stimulus occurred 50 or $100 \mathrm{~ms}$ before $(0.11 \pm 0.04$ bits for ${ }_{p w}^{50} P W$ vs ${ }_{s w}^{50} P W ; 0.10 \pm 0.04$ bits for ${ }_{p w}^{100} P W$ vs ${ }_{s w}^{100} P W$ ), or if there was uncertainty for the decoder about the timing of the previous stimulus $\left(0.08 \pm 0.03\right.$ bits for ${ }_{p w}^{50 \mid 100} P W$ vs. $\left.{ }_{s w}^{50 \mid 100} P W\right)$. This result suggests a consistent capacity of the adapted PW response to discriminate the spatial context of the previous stimulus, at least within $100 \mathrm{~ms}$.

We then examined the capacity of the adapted PW response to the current stimulus to discriminate when the previous stimulus occurred, 50 or $100 \mathrm{~ms}$ in the past (Fig. 4D). The adapted PW response provided significantly more information if the previous stimulus occurred in the same location as the current stimulus $\left({ }_{p w}^{50} P W\right.$ vs ${ }_{p w}^{100} P W, 0.10 \pm 0.05$ bits $)$, than if the previous stimulus occurred in the different location ${ }_{s w}^{50} S W$ vs ${ }_{s w}^{100} S W, 0.03 \pm 0.01$ bits, $\left.t_{(39)}=2.86, p=0.0067\right)$. The capacity of the adapted PW response to discriminate the temporal context of the previous stimulus was thus dependent on its spatial context.

To corroborate that the adapted PW response conveys both the spatial and temporal contextual information about a stimulus in the past, we examined the capacity of the adapted PW response to the current stimulus to simultaneously discriminate both where and when the previous stimulus occurred in the past ${ }_{p w}^{50} P W$ vs ${ }_{p w}^{100} P W$ vs ${ }_{s w}^{50} P W$ vs $\left.{ }_{s w}^{100} P W\right)$. We could extract $0.14 \pm 0.04$ bits of information, corroborating that adapted responses convey both spatial and temporal information about previous stimuli. 
A
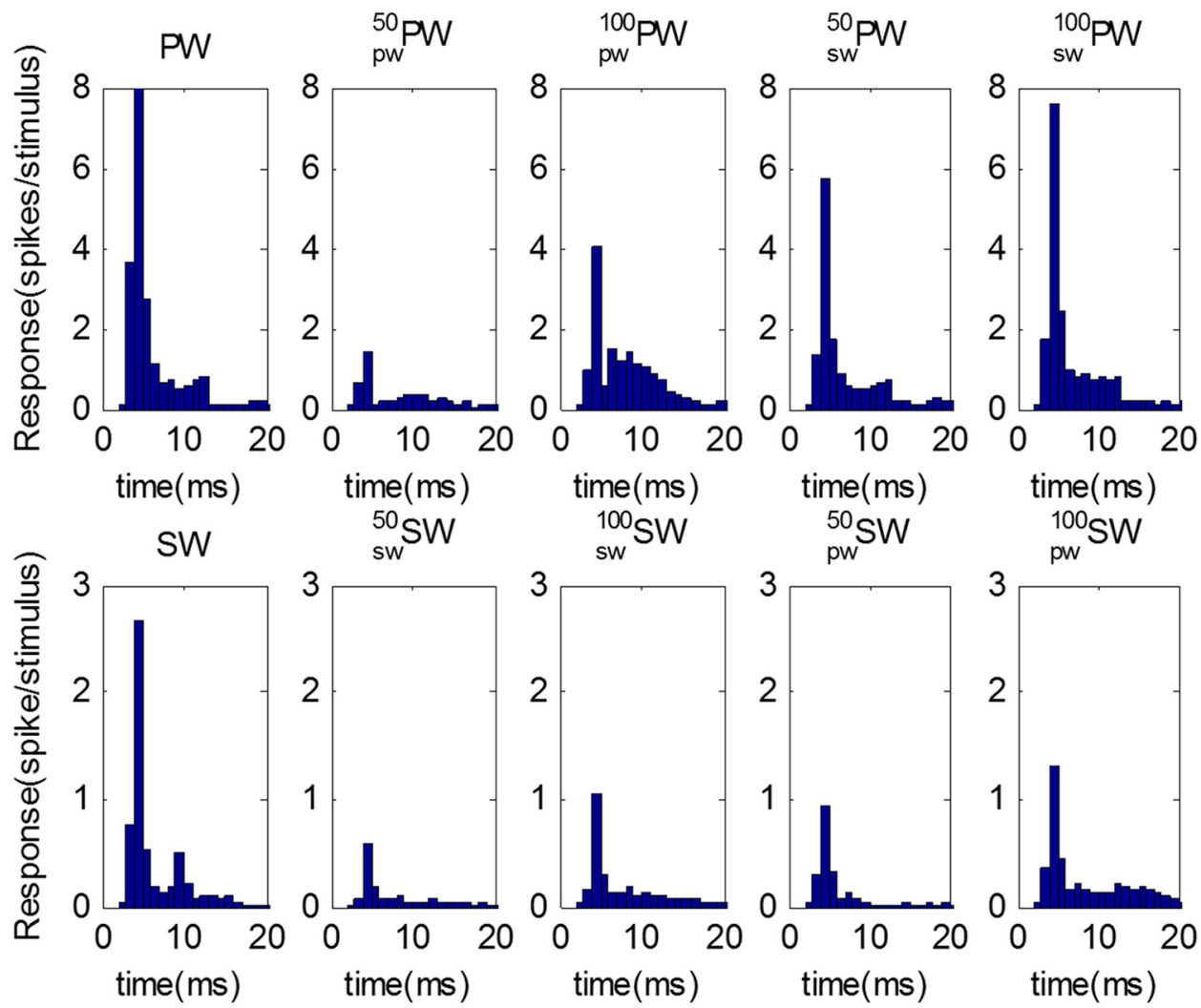

B
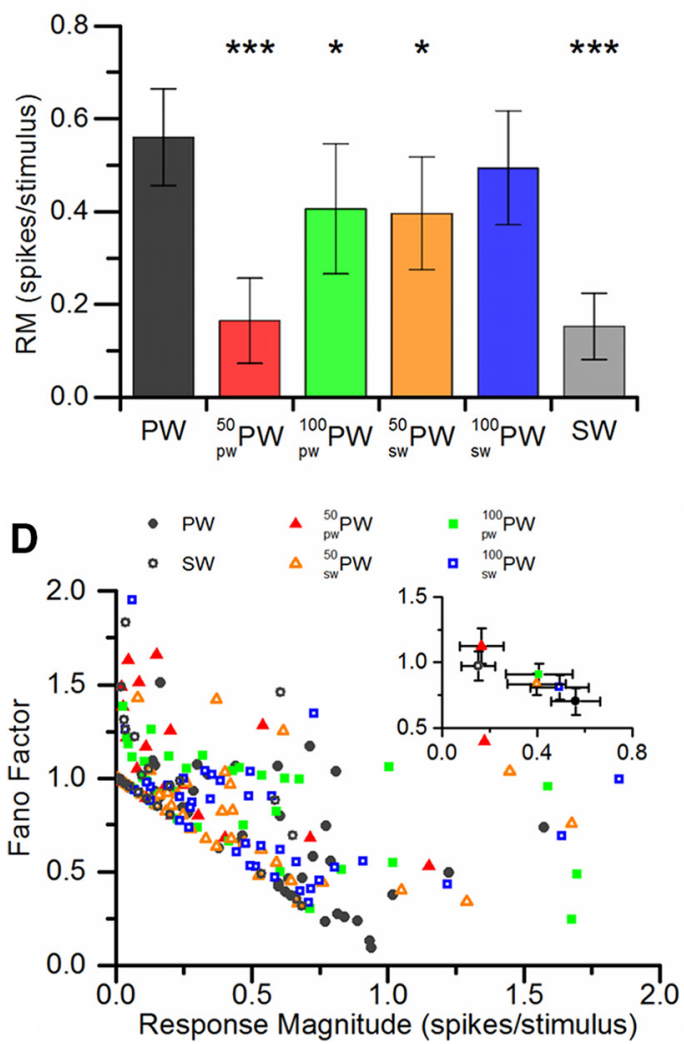

C
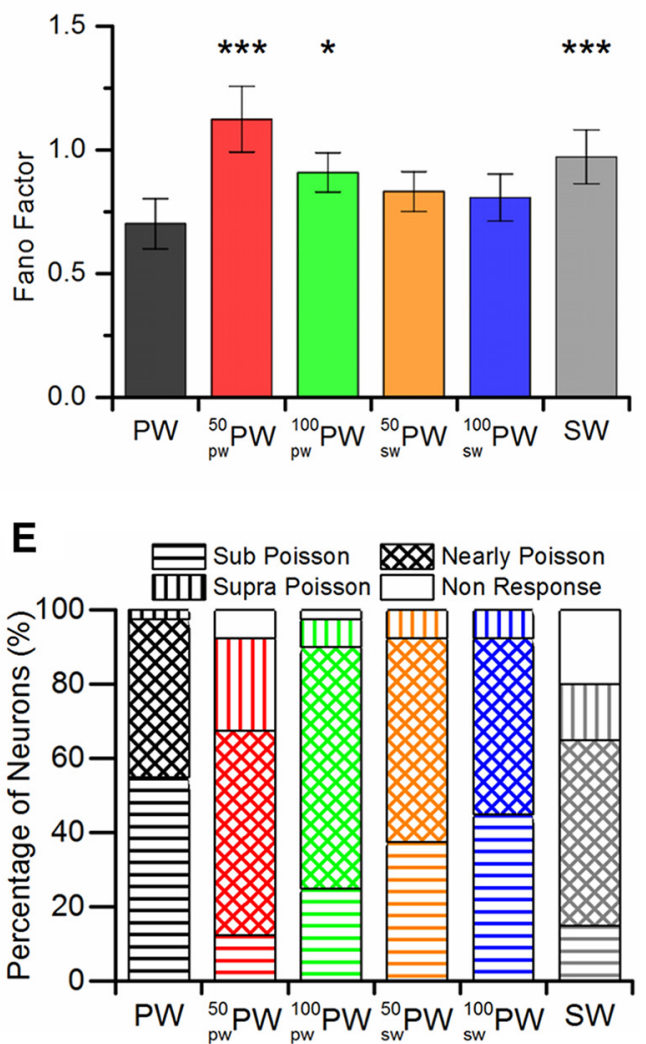

Figure 2. Neurophysiological properties of VPM neurons to paired-pulse stimuli. $\boldsymbol{A}$, Population responses of all 40 neurons; the top plots represent the population PSTH for the five groups of PW responses, whereas bottom plots represent the population PSTH for the five groups of SW responses. $\boldsymbol{B}$, Response magnitude (RM) and ( $\boldsymbol{C}$ ) FF for the five groups of PW responses and the non-adapted SW response. The black stars indicate the significance levels for the post hoc Dunnett test for each adapted PW response and non-adapted SW response relative to the non-adapted PW response. ${ }^{*} p<0.05,{ }^{* *} p<0.01,{ }^{* * *} p<0.001$. D, Scatter plot of FF versus RM. Inset, The average FF and RM of the VPM neurons in each group. $\boldsymbol{E}$, The proportion of sub-Poisson, nearly Poisson, and supra-Poisson cells based on FF measurements in each group of PW responses. Error bars represent $95 \%$ confidence intervals. 
A

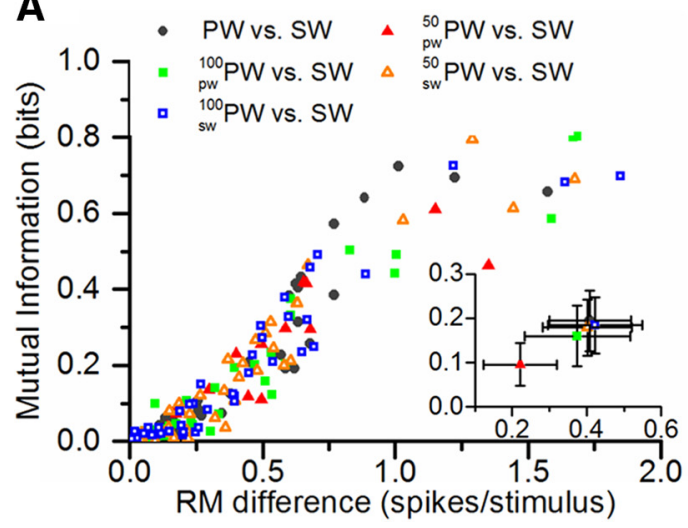

B

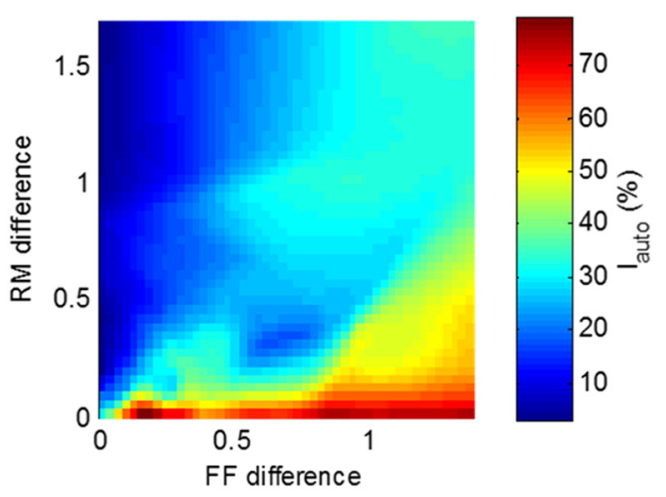

Figure 3. Information about stimulus location in the present carried by single neurons. $A$, Scatter plot of mutual information versus RM difference between PW stimulus (non-adapted or adapted) and SW stimulus. Inset, The average mutual information and RM difference for each group of adapted PW responses. B, Color map of percentage of autocorrelation information (\% of total information), dependent on RM difference and FF difference between PW and SW stimulus. Error bars represent $95 \%$ confidence intervals.

A

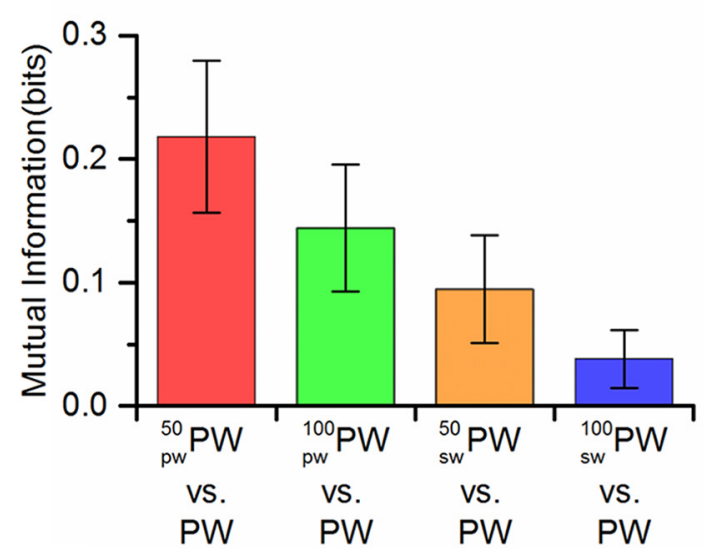

C

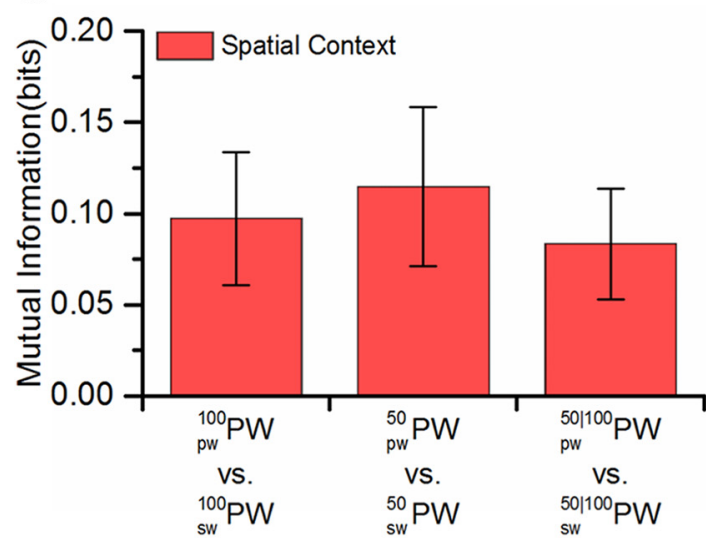

B

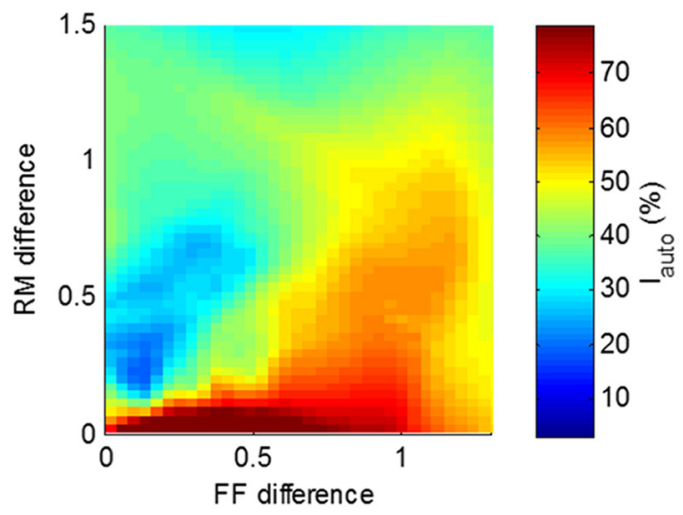

D

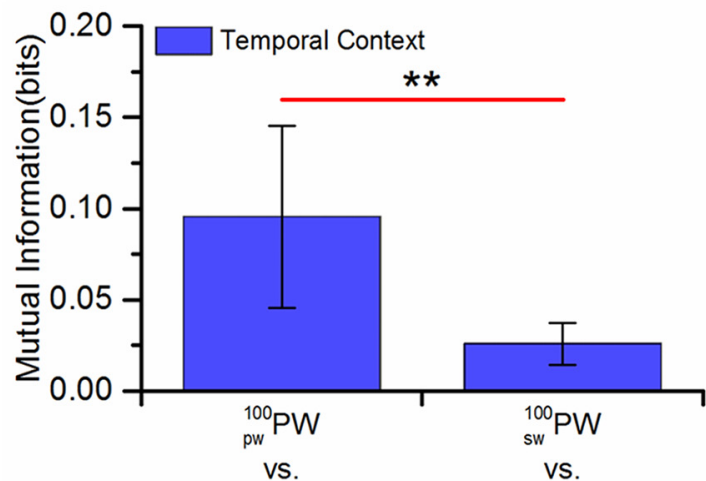

${ }_{\mathrm{pw}}^{50} \mathrm{PW}$
VS.

${ }_{\text {sw }}^{50} \mathrm{PW}$

Figure 4. Information about stimulus history carried by single neurons. $A$, Mutual information for detecting whether a prior stimulus occurred, (B) Color map of percentage of autocorrelation information (\% of total information) as a function of RM and FF differences between the adapted and non-adapted PW stimulus. The result is similar to Figure $3 B$. C, Mutual information to discriminate spatial context ${ }_{\mathrm{pw}} \mathrm{PW}$ vs ${ }_{\text {sw }} \mathrm{PW}$; red) and (D) temporal context (100 or $50 \mathrm{~ms}$ prior; blue) of the occurred previous stimulus. Error bars represent $95 \%$ confidence intervals. ${ }^{* *} p<0.01$.

Effect of adaptation on the capacity of populations of neurons to convey information

So far we showed that adaptation allows single neurons to gain information about whether, where and when a previous stimulus occurred, but this comes at the expense of a loss of information about the current stimulus. To test whether this loss of information could be compensated at the population level, we measured the information of pooled neuronal responses when discriminating current stimulus location (Fig. 5).

When discriminating current stimulus location from nonadapted responses (i.e., PW vs SW), the more neurons used in the discrimination the greater the information, reaching the limit of 1 bit for the binary classification problem. Using the knowledge of the identities of the neurons with the PSTH decoder (i.e., ex- 
A

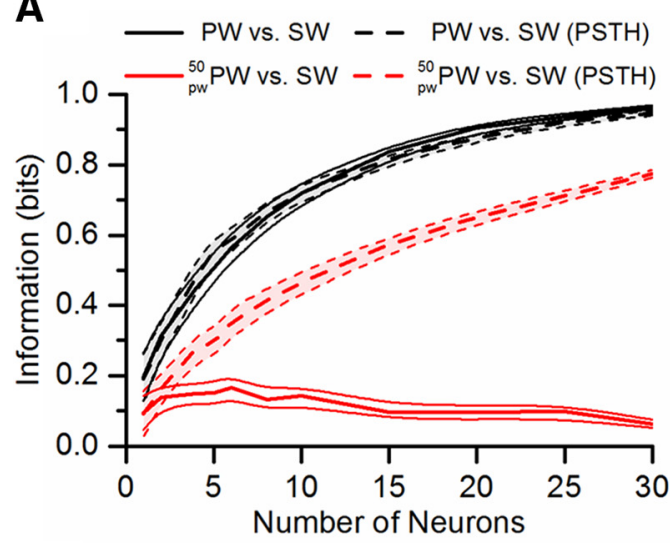

C

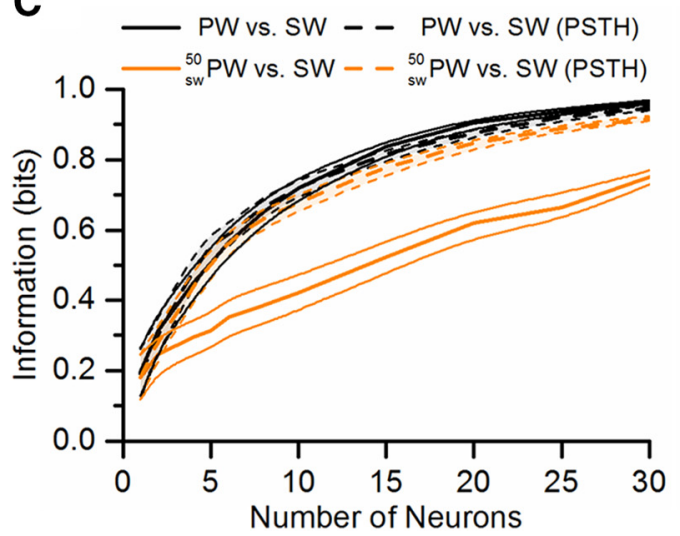

B

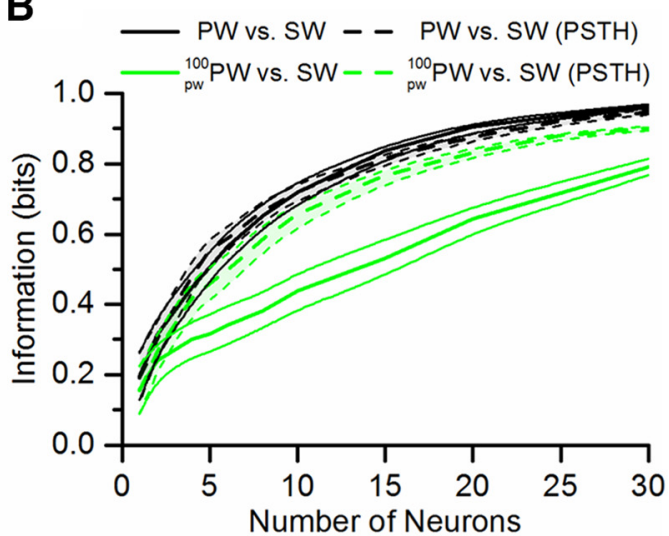

D

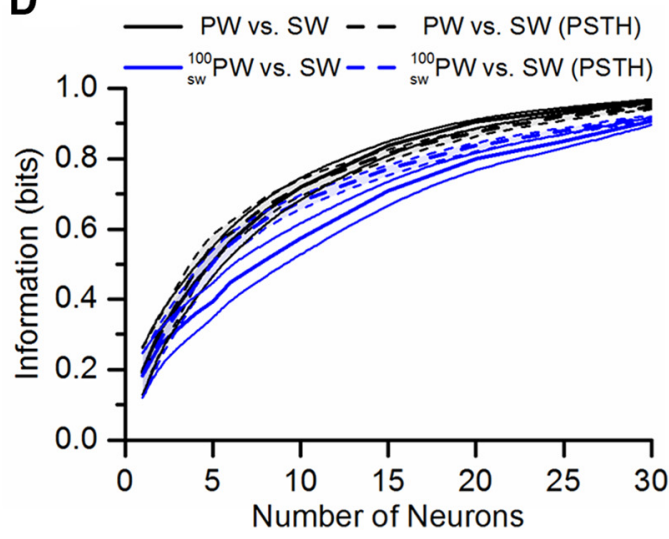

Figure 5. Information about stimulus location in the present carried by total number of spikes (solid line) or population response decoded with PSTH classification preserving neuron identities (dashed line). Information values are obtained from 100 permutations of random sampling of neurons. Boundary lines (light solid or light dashed) indicate $95 \%$ confidence intervals. $\boldsymbol{A}, \boldsymbol{B}$, Discriminating between adapted PW responses and SW response when the previous stimulus was the PW, $50 \mathrm{~ms}$ earlier $(\boldsymbol{A})$ or $100 \mathrm{~ms}$ earlier $(\boldsymbol{B})$. $\boldsymbol{C}, \boldsymbol{D}$, Discriminating between the adapted PW response and the SW response when the previous stimulus was SW, 50 ms earlier $(\boldsymbol{C})$ or $100 \mathrm{~ms}$ earlier $(\boldsymbol{D})$.

ploiting the heterogeneity among neurons) did not add additional information compared with simply using the total number of spikes produced by the population (Fig. $5 A$ ). This was due to the fact that non-adapted PW responses were homogeneous, in the sense that they were generally much greater than non-adapted SW responses. However, adaptation disrupted this relationship, rendering the heterogeneity among neurons particularly informative. Consequently, when discriminating between adapted PW responses and SW responses (e.g., ${ }_{p w} P W$ vs SW; Fig. $5 A$ ), knowledge of the neurons' identities became critical for information to increase as the number of neurons increases. When adaptation was less severe [e.g., ${ }_{p w}^{100} P W$ vs SW (Fig. $5 B$ ) or ${ }_{p w}^{50} P W$ vs PW (Fig. $5 C$ )], the responses remained more homogeneous, so more neurons contributed more information even if knowledge of the neurons' identities were not used. Yet, knowing the identities of the individual neurons by using the PSTH classifier allowed for more information to be decoded than simply pooling the response magnitude of neurons. It was not until there was negligible adaptation (e.g., ${ }_{s w}^{100} \mathrm{PW}$ vs SW; Fig. 5D) that the information extracted was similar to the non-adapted case and there was no loss of information if the identities of neurons were not used. Interestingly, information per spike increased in the adapted compared with the non-adapted case, but only when the knowledge of neuron identity was considered (Fig. 6). Therefore, when discriminating an adapted response, an ideal decoder can compensate for the decrease in the response magnitude by using the responses of population neurons.

\section{Discussion}

The results of the present work confirm our intuitive understanding that the physiological changes (i.e., attenuation of response magnitude and increased response variability) induced by pairedpulse adaptation increase the ambiguity of discriminating stimulus location at the single-neuron level. However, the adapted responses are capable of carrying significant contextual information about whether, when and where there was a stimulus in the past. Importantly, the ambiguity about the present stimulus is tempered by increasing the number of recruited neurons, especially if the downstream decoder maintains the identity of the neurons to exploit interneuron heterogeneity. Furthermore, information per spike is higher in the adapted compared with the nonadapted state. These results therefore suggest that the process of adaptation allows thalamic neurons to integrate a rich spatial and temporal context of the sensory stimulus history, whereas the population response ensures reliable processing of simple stimuli.

\section{Methodological considerations}

The impact of neural adaptation is well established as a reduction in response magnitude of single neurons during consecutive presentation of a stimulus. Prolonged repetitive stimulation induces a steady-state adaptation of thalamocortical responses (Deschênes et al., 2003; Hartings et al., 2003; Khatri et al., 2004; Aguilar and CastroAlamancos, 2005), which results from a dynamic equilibrium of accumulated short-term effects, involving bottom-up, local, and 
A

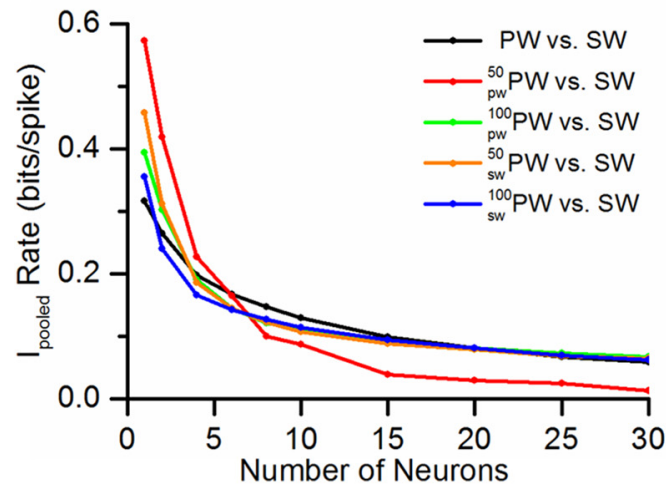

B

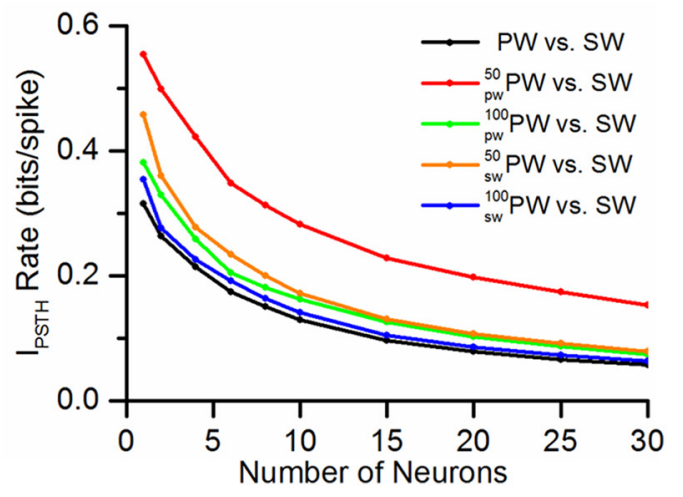

Figure 6. Information rate (i.e., bits per spike) about stimulus location in the present carried by $(\boldsymbol{A})$ total number of spikes or (B) population response decoded with PSTH classification preserving neuron identities.

top-down mechanisms (Lampl and Katz, 2017), with an important contribution of corticothalamic feedback (Canedo and Aguilar, 2000; Temereanca and Simons, 2004; Hirata et al., 2006). To exclude accumulation of short-term effects, here we focused on paired-pulse adaptation. The degree of paired-pulse adaptation depended on two parameters of the stimulus that are relevant for active whisking: stimulus frequency (i.e., 50 vs $100 \mathrm{~ms}$ interstimulus interval) and stimulus location (i.e., same-whisker vs cross-whisker stimulus; Bosman et al., 2011; McDonald et al., 2014). There are clearly other parameters of stimulation (e.g., magnitude of stimulation) that still require investigation.

Electrical stimulation may activate the innervating axons of the follicles differently than mechanical stimulation. For example, rapidly-adapting and slowly adapting fibers may be activated indiscriminately using electrical stimulation, whereas mechanical stimulation is likely to recruit each population in a more natural order related to the position of the nerve endings within the follicle and the direction of the whisker movement. Nevertheless, it has been showed that electrical and mechanical stimulation delivered to the targeted whisker are virtually indistinguishable (Devilbiss and Waterhouse, 2002). In our experiments, when the PW was stimulated, the responses of neurons were slightly smaller than the typical responses of VPM neurons to mechanical stimulation of the principal whisker during a quiescent state $(0.7 \pm 0.1$ spikes/stimulus with $<7$ ms latency; Aguilar and Castro-Alamancos, 2005). Even though we cannot exclude that the primary whisker was not the principal whisker in some cells, these response properties suggest that our electrical stimulation reasonably mimics the response expected from mechanical stimulation of the whisker.

In our experiment only one SW was stimulated. Therefore, we do not have data to generalize the information affected by adaptation to the problem of discriminating between multiple adjacent whiskers, possibly eliciting similar response magnitudes. However, we show that trial-to-trial variability is modulated by adaptation and that when response magnitudes are similar the difference in trial-to-trial variability between stimuli becomes particularly important for stimulus discrimination (Scaglione et al., 2011). Similarly, response latency is also modulated by adaptation, and we know that differences in response latency, and thus spike timing, become particularly informative when response magnitudes are similar (Foffani et al., 2008, 2009). Therefore, trial-to-trial variability and spike timing are also likely to provide an important contribution to the information about the spatiotemporal context of stimulus history, particularly in more complex paradigms.
Our information analyses about the ability of adapted singleneuron responses to discriminate whether, where, and when a stimulus occurred in the past are based on PW responses. This implicitly assumes that the decoder knows (1) that there is indeed a present stimulus and (2) that the response is a PW response. The first assumption seems reasonable because stimulus detection is a relatively easy computational task compared with stimulus discrimination (Foffani et al., 2008). The second assumption is somewhat trickier, but essentially implies that the decoder can discriminate PW responses from SW responses (which is supported by our results), and spatially confines the information about past stimuli within the PW barreloid. Future work should establish whether and how this information is redundantly parallelized across barreloids, possibly exploiting latency differences and spike timing (Foffani et al., 2008, 2009).

It is also important to note that, when addressing the role of population coding, the neurons were pooled and not recorded simultaneously. Pooled responses from populations of non-simultaneously reordered neurons exclude information contributed by "noise-correlations" between neurons, but this contribution seems to be negligible in our experimental conditions (Scaglione et al., 2011). Moreover, adaptation tends to decorrelate the response of cells, making the signals less redundant (Barlow and Földiák, 1989; Kohn, 2007; Khatri et al., 2009; Wissig and Kohn, 2012; but see, Adibi et al., 2013a). Therefore, our conclusion that increasing the number of recruited numbers of neurons compensates for the loss of information about the current stimulus is likely to be conservative.

Last, our study used passive whisker stimulation in the anesthetized rat. The advantage of this approach is that we have relatively complete control of the stimuli across conditions (Kohn and Movshon, 2004) and it is known that awake rats can discriminate passive stimuli (Kleinfeld and Deschênes, 2011). However, further work is required to extend our findings to more complex stimuli, to natural exploratory behaviors and to different behavior/attentive states (Aguilar and Castro-Alamancos, 2005; Gerdjikov et al., 2010; Stüttgen, 2010; Scaglione et al., 2014).

\section{The adapted response carries information about stimulus history, whereas population coding ensures reliable discrimination of the current stimulus}

Although information conveyed by single neurons about the current stimulus is reduced, adaptation allows an ideal decoder to gain information about whether, where, and when there was a 
stimulus in the recent past. Adaptation may thus provide information about the spatiotemporal context of stimulus history.

The loss of information imposed by adaptation about the current stimulus seems an unavoidable tradeoff to gain information about the short-term history of the stimuli. But the tradeoff may not be of significance when one considers population coding. In fact, our results reveal that with large numbers of neurons the loss of information about the current stimulus due to adaptation may become negligible. This is particularly true if the ideal decoder has knowledge of neuron identity to exploit the interneuron heterogeneity, which is an implicit assumption made by most studies on population coding. Indeed, the cortex can read out stable information from the heterogeneity and diversity of the ascending pathways (Vinje and Gallant, 2000; Olshausen and Field, 2004; Farkhooi et al., 2013).

Our results also show that paired-pulse adaptation increases information per spike. This is in good agreement with recent findings reporting increased spike efficiency with adaptation in the rat barrel cortex (Adibi et al., 2012; 2013a) and with the general optimization of information transmission observed with other adaptation paradigms (Barlow, 1961; Fairhall et al., 2001; Dean et al., 2005; Maravall et al., 2007). It is tempting to suggest that increased information per spike may imply lower metabolic cost for spiking, but this should be verified taking into account all local, bottom-up and top-down contributions to VPM adaptation (Lampl and Katz, 2017). In any case, our data suggest that adaptation may actually increase the efficiency of information transmission about the present stimulus, while providing the spatiotemporal context of stimulus history.

The possible impact of this information on cortical processing is intriguing. It was recently shown in the barrel cortex that information about the time of the previous stimulus is maintained across all cortical layers (Pitas et al., 2017), with deep layers that receive direct thalamic input having the least amount of temporal integration and the supragranular layers having substantially more. Together with our understanding of the transformation of adaptation from brainstem to thalamus (Ganmor et al., 2010; Mohar et al., 2015; Jubran et al., 2016), these data suggest that there is both local and inherited processing of stimulus history at each level of the whisky sensory system.

Finally, it has been proposed that neuronal networks are inherently capable of processing complex spatiotemporal stimuli as a result of interactions between an external stimulus and an internal, "hidden state" of the network (Buonomano and Maass, 2009; Klampfl et al., 2012). From this view, it is interesting to propose that changes in synaptic and cellular properties induced by adaptation represent a change in the hidden state of the network that maintains information about previous stimuli while allowing the network to respond to subsequent stimuli. Our study expands this possibility by quantifying the spatiotemporal information about past stimuli that can be maintained by the network, and supporting the idea that internal network states exist, at least in part, in subcortical networks such as thalamus.

Overall, our results suggest that at the population level adapted responses do not lose the ability to discriminate stimulus location, they increase spike efficiency, and they gain the capacity to provide information about the spatiotemporal short-term history of the stimuli. Therefore, adaptation may be an efficient coding scheme to optimally encode ongoing stimuli while tracking stimulus history, thereby maximizing information to achieve a sensory task.

\section{References}

Adibi M, Diamond ME, Arabzadeh E (2012) Behavioral study of whiskermediated vibration sensation in rats. Proc Natl Acad Sci U S A 109:971976. CrossRef Medline

Adibi M, McDonald JS, Clifford CW, Arabzadeh E (2013a) Adaptation improves neural coding efficiency despite increasing correlations in variability. J Neurosci 33:2108-2120. CrossRef Medline

Adibi M, Clifford CW, Arabzadeh E (2013b) Informational basis of sensory adaptation: entropy and single-spike efficiency in rat barrel cortex. J Neurosci 33:14921-14926. CrossRef Medline

Aguilar JR, Castro-Alamancos MA (2005) Spatiotemporal gating of sensory inputs in thalamus during quiescent and activated states. J Neurosci 25: 10990-11002. CrossRef Medline

Anderson LA, Christianson GB, Linden JF (2009) Stimulus-specific adaptation occurs in the auditory thalamus. J Neurosci 29:7359-7363. CrossRef Medline

Barlow HB (1961) Possible principles underlying the transformation of sensory messages. In: Sensory communication (Rosenblith W, ed), pp 217234. Cambridge, MA: MIT.

Barlow HB, Földiák P (1989) Adaptation and decorrelation in the cortex. In: Computing neuron (Durbin R, Miall C, Mitchison G, eds), pp 54-72. Boston: Addison-Wesley.

Bosman LW, Houweling AR, Owens CB, Tanke N, Shevchouk OT, Rahmati N, Teunissen WH, Ju C, Gong W, Koekkoek SK, De Zeeuw CI (2011) Anatomical pathways involved in generating and sensing rhythmic whisker movements. Front Integr Neurosci 5:53. CrossRef Medline

Buonomano DV, Maass W (2009) State-dependent computations: spatiotemporal processing in cortical networks. Nat Rev Neurosci 10:113-125. CrossRef Medline

Butts DA, Goldman MS (2006) Tuning curves, neuronal variability, and sensory coding. PLoS Biol 4:e92. CrossRef Medline

Canedo A, Aguilar J (2000) Spatial and cortical influences exerted on cuneothalamic and thalamocortical neurons of the cat. Eur J Neurosci 12:25152533. CrossRef Medline

Castro-Alamancos MA (2004) Dynamics of sensory thalamocortical synaptic networks during information processing states. Prog Neurobiol 74: 213-247. CrossRef Medline

Chase SM, Kass RE, Schwartz AB (2012) Behavioral and neural correlates of visuomotor adaptation observed through a brain-computer interface in primary motor cortex. J Neurophysiol 108:624-644. CrossRef Medline

Chung S, Li X, Nelson SB (2002) Short-term depression at thalamocortical synapses contributes to rapid adaptation of cortical sensory responses in vivo. Neuron 34:437-446. CrossRef Medline

Dean I, Harper NS, McAlpine D (2005) Neural population coding of sound level adapts to stimulus statistics. Nat Neurosci 8:1684-1689. CrossRef Medline

Deschênes M, Timofeeva E, Lavallée P (2003) The relay of high-frequency sensory signals in the whisker-to-barreloid pathway. J Neurosci 23:67786787. Medline

Devilbiss DM, Waterhouse BD (2002) Determination and quantification of pharmacological, physiological, or behavioral manipulations on ensembles of simultaneously recorded neurons in functionally related neural circuits. J Neurosci Methods 121:181-198. CrossRef Medline

Eden UT, Kramer MA (2010) Drawing inferences from Fano factor calculations. J Neurosci Methods 190:149-152. CrossRef Medline

Fairhall AL, Lewen GD, Bialek W, de Ruyter Van Steveninck RR (2001) Efficiency and ambiguity in an adaptive neural code. Nature 412:787-792. CrossRef Medline

Farkhooi F, Froese A, Muller E, Menzel R, Nawrot MP (2013) Cellular adaptation facilitates sparse and reliable coding in sensory pathways. PLoS Comput Biol 9:e1003251. CrossRef Medline

Foffani G, Moxon KA (2004) PSTH-based classification of sensory stimuli using ensembles of single neurons. J Neurosci Methods 135:107-120. CrossRef Medline

Foffani G, Tutunculer B, Moxon KA (2004) Role of spike timing in the forelimb somatosensory cortex of the rat. J Neurosci 24:7266-7271. CrossRef Medline

Foffani G, Chapin JK, Moxon KA (2008) Computational role of large receptive fields in the primary somatosensory cortex. J Neurophysiol 100:268280. CrossRef Medline

Foffani G, Morales-Botello ML, Aguilar J (2009) Spike timing, spike count, 
and temporal information for the discrimination of tactile stimuli in the rat ventrobasal complex. J Neurosci 29:5964-5973. CrossRef Medline

Ganmor E, Katz Y, Lampl I (2010) Intensity-dependent adaptation of cortical and thalamic neurons is controlled by brainstem circuits of the sensory pathway. Neuron 66:273-286. CrossRef Medline

Gerdjikov TV, Bergner CG, Stüttgen MC, Waiblinger C, Schwarz C (2010) Discrimination of vibrotactile stimuli in the rat whisker system: behavior and neurometrics. Neuron 65:530-540. CrossRef Medline

Gutnisky DA, Dragoi V (2008) Adaptive coding of visual information in neural populations. Nature 452:220-224. CrossRef Medline

Haith A, Jackson CP, Miall RC, Vijayakumar S (2009) Unifying the sensory and motor components of sensorimotor adaptation. In: Advances in Neural Information Processing Systems 21-Proceedings of the 2008 Conference, pp 593-600. Vancouver, BC: 22nd Annual Conference on Neural Information Processing Systems.

Hartings JA, Temereanca S, Simons DJ (2003) Processing of periodic whisker deflections by neurons in the ventroposterior medial and thalamic reticular nuclei. J Neurophysiol 90:3087-3094. CrossRef Medline

Higley MJ, Contreras D (2005) Integration of synaptic responses to neighboring whiskers in rat barrel cortex in vivo. J Neurophysiol 93:1920-1934. CrossRef Medline

Higley MJ, Contreras D (2007) Cellular mechanisms of suppressive interactions between somatosensory responses in vivo. J Neurophysiol 97:647658. CrossRef Medline

Hirata A, Aguilar J, Castro-Alamancos MA (2006) Noradrenergic activation amplifies bottom-up and top-down signal-to-noise ratios in sensory thalamus. J Neurosci 26:4426-4436. CrossRef Medline

Hollins M, Harper D, Maixner W (2011) Changes in pain from a repetitive thermal stimulus: the roles of adaptation and sensitization. Pain 152: 1583-1590. CrossRef Medline

Jubran M, Mohar B, Lampl I (2016) The transformation of adaptation specificity to whisker identity from brainstem to thalamus. Front Syst Neurosci 10:56. CrossRef Medline

Khatri V, Hartings JA, Simons DJ (2004) Adaptation in thalamic barreloid and cortical barrel neurons to periodic whisker deflections varying in frequency and velocity. J Neurophysiol 92:3244-3254. CrossRef Medline

Khatri V, Bruno RM, Simons DJ (2009) Stimulus-specific and stimulusnonspecific firing synchrony and its modulation by sensory adaptation in the whisker-to-barrel pathway. J Neurophysiol 101:2328-2338. CrossRef Medline

Klampfl S, David SV, Yin P, Shamma SA, Maass W (2012) A quantitative analysis of information about past and present stimuli encoded by spikes of A1 neurons. J Neurophysiol 108:1366-1380. CrossRef Medline

Kleinfeld D, Deschênes M (2011) Neuronal basis for object location in the vibrissa scanning sensorimotor system. Neuron 72:455-468. CrossRef Medline

Kohn A (2007) Visual adaptation: physiology, mechanisms, and functional benefits. J Neurophysiol 97:3155-3164. CrossRef Medline

Kohn A, Movshon JA (2004) Adaptation changes the direction tuning of macaque MT neurons. Nat Neurosci 7:764-772. CrossRef Medline

Lampl I, Katz Y (2017) Neuronal adaptation in the somatosensory system of rodents. Neuroscience 343:66-76. CrossRef Medline

Laughlin SB (1989) The role of sensory adaptation in the retina. J Exp Biol 146:39-62. Medline
Lavallée P, Deschênes M (2004) Dendroarchitecture and lateral inhibition in thalamic barreloids. J Neurosci 24:6098-6105. CrossRef Medline

Magri C, Whittingstall K, Singh V, Logothetis NK, Panzeri S (2009) A toolbox for the fast information analysis of multiple-site LFP, EEG and spike train recordings. BMC Neurosci 10:81. CrossRef Medline

Maravall M, Petersen RS, Fairhall AL, Arabzadeh E, Diamond ME (2007) Shifts in coding properties and maintenance of information transmission during adaptation in barrel cortex. PLoS Biol 5:e19. CrossRef Medline

McDonald JS, Adibi M, Clifford CW, Arabzadeh E (2014) Sampling time and performance in rat whisker sensory system. PLoS One 9:e116357. CrossRef Medline

Mohar B, Ganmor E, Lampl I (2015) Faithful representation of tactile intensity under different contexts emerges from the distinct adaptive properties of the first somatosensory relay stations. J Neurosci 35:69977002. CrossRef Medline

Moxon KA, Hallman S, Sundarakrishnan A, Wheatley M, Nissanov J, Barbee KA (2009) Long-term recordings of multiple, single-neurons for clinical applications: the emerging role of the bioactive microelectrode. Materials 2:1762-1794. CrossRef

Nelken I, Chechik G, Mrsic-Flogel TD, King AJ, Schnupp JW (2005) Encoding stimulus information by spike numbers and mean response time in primary auditory cortex. J Comput Neurosci 19:199-221. CrossRef Medline

Olshausen BA, Field DJ (2004) Sparse coding of sensory inputs. Curr Opin Neurobiol 14:481-487. CrossRef Medline

Pitas A, Albarracín AL, Molano-Mazón M, Maravall M (2017) Variable temporal integration of stimulus patterns in the mouse barrel cortex. Cereb Cortex 27:1758-1764. CrossRef Medline

Ranganath C, Rainer G (2003) Neural mechanisms for detecting and remembering novel events. Nat Rev Neurosci 4:193-202. CrossRef Medline

Scaglione A, Moxon KA, Foffani G (2010) General Poisson exact breakdown of the mutual information to study the role of correlations in populations of neurons. Neural Comput 22:1445-1467. CrossRef Medline

Scaglione A, Moxon KA, Aguilar J, Foffani G (2011) Trial-to-trial variability in the responses of neurons carries information about stimulus location in the rat whisker thalamus. Proc Natl Acad Sci U S A 108:14956-14961. CrossRef Medline

Scaglione A, Foffani G, Moxon KA (2014) Spike count, spike timing and temporal information in the cortex of awake, freely moving rats. J Neural Eng 11:046022. CrossRef Medline

Stüttgen MC (2010) Toward behavioral benchmarks for whisker-related sensory processing. J Neurosci 30:4827-4829. CrossRef Medline

Stüttgen MC, Schwarz C (2010) Integration of vibrotactile signals for whisker-related perception in rats is governed by short time constants: comparison of neurometric and psychometric detection performance. J Neurosci 30:2060-2069. CrossRef Medline

Temereanca S, Simons DJ (2004) Functional topography of corticothalamic feedback enhances thalamic spatial response tuning in the somatosensory whisker/barrel system. Neuron 41:639-651. CrossRef Medline

Vinje WE, Gallant JL (2000) Sparse coding and decorrelation in primary visual cortex during natural vision. Science 287:1273-1276. CrossRef Medline

Wark B, Lundstrom BN, Fairhall A (2007) Sensory adaptation. Curr Opin Neurobiol 17:423-429. CrossRef Medline

Wissig SC, Kohn A (2012) The influence of surround suppression on adaptation effects in primary visual cortex. J Neurophysiol 107:3370-3384. CrossRef Medline 